\section{Factores de la dinámica famililar de los niños y niñas en situación de calle en arededores del Mercado Mayorista que determinan su expulsión del seno familiar}

\author{
Factors in the family dynamic of children in streets around \\ Mayorista Market that determine their expulsion from \\ the family
}

\section{RESUMEN}

Objetivos: La investigación fue para determinar los factores de la dinámica familiar de los niños y niñas en situación de calle en alrededores del Mercado Mayorista que propiciaron su expulsión del seno familiar. Métodos: Es una investigación de tipo básica, de nivel descriptivo; de carácter cualitativo; el método que se utilizó fue el análisis síntesis, y una muestra no probabilística, se desarrolló a partir del estudio de caso de cinco niños que viven en la calle en alrededores del Mercado Mayorista de la ciudad de Huancayo, Perú; que fueron identificados a partir de informantes claves, se hizo abordaje paulatinos y progresivos y cuando ya se estableció un nivel mínimo de confianza, se les registró su testimonio. Resultados: Los factores de la dinámica familiar que determinan la expulsión del seno familiar son la desestructuración en su cohesión familiar no solo por producto de ruptura conyugal; sino también por dos características identificadas en esta población uno es el proceso de "migración" y otro es "las experiencias de violencia" que se dan al interior del seno familiar; otros factores de la dinámica familiar son: la existencia de inadecuados niveles de comunicación familiar producto de la incompatibilidad de caracteres entre padres e hijos y por la existencia de un negativo clima emocional afectivo por las escasas expresiones de afecto entre padres e hijos. Conclusiones: Se encontró que son niños y no niñas que se ven afectados por los problemas al interior de la familia y los principales factores para determinar su expulsión son el proceso de "migración" y otro es "las experiencias de violencia"; así mismo los inadecuados niveles de comunicación familiar y la existencia de un clima emocional afectivo.

Palabras clave: Niño, familia, afecto, dinámica familiar.

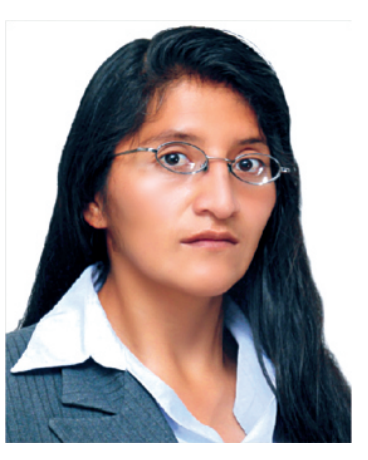

Cecilia G. Aliaga Sandoval Universidad Nacional del Centro del Perú

\section{ABSTRACT}

Objectives: The research was to determine the factors of the family dynamic of children in streets around Mayorista Market that propitiated their expulsion from the family. Methods: It is a investigation of basic type, descriptive level; qualitative character; the method that was applied was the analysis - synthesis, and non probabilistic sample, it was developed from the study of five children's case that live in the street around Mayorista Market in Huancayo city, those children were identified from key informants, gradual and progressive approaching was made and when we already had a minimum level of trust or confidence we were taken their testimony. Results: The family dynamic factors that determine the expulsion from family are the

1 Lic. en Trabajo Social, egresada de la Maestría de Políticas Sociales con mención en Infancia, de la Universidad Nacional del Centro del Perú. 
disintegration in family cohesion not only as product for marital breakdown but also by two identified characteristics in this population, one is a process of "migration" and another is "the experiences of violence" that happened inside in the family", other family factors are the existence of inadequate levels of family communication which is product of the incompatibility between parents and children's characthers; also, the existence of a negative kindness and emotional environment because of the lack of kind expressions between parents and children. Conclusions: It was found that boys and not girls are affected by the problems inside of the family, the main factors to determine their expulsion are the "migration" process and another "experiences of violence," likewise inadequate levels of family communication and the existence of an affective emotional environment.

Key words: Child, family, affection, family dynamic.

\section{INTRODUCCIÓN}

La investigación realizada no tiene antecedentes locales ni regionales. Mi conocimiento y experiencia de trabajo en temas de infancia motivaron para realizar esta investigación con este tipo de población olvidada y excluida tanto por autoridades locales y el gobierno central; partió de la siguiente interrogante: ¿̇Qué factores de la dinámica familiar de los niños y niñas en situación de calle en alrededores del Mercado Mayorista determinan su expulsión del seno familiar?; así mismo se planteó como hipótesis lo siguiente: "los factores de la dinámica familiar de los niños y niñas en situación de calle en alrededores del Mercado Mayorista que determinan su expulsión del seno familiar son la desestructuración en su cohesión, inadecuados niveles de comunicación familiar y un negativo clima emocional afectivo", hipótesis que fue confirmada y contrastada con la realidad estudiada; el objetivo planteado fue: "describir los factores de la dinámica familiar de los niños y niñas en situación de calle en alrededores del Mercado Mayorista que determinan su expulsión del seno familiar"; para sustentar nuestra investigación utilizamos las siguientes teorías científicas: la Teoría del Rol, la Teoría General de Sistemas y la Teoría de la Acción Social de Parson.

\section{MATERIAL Y MÉTODOS}

Es una investigación de tipo básica, de nivel descriptivo; de carácter cualitativo; el método que se utilizó fue el análisis síntesis: se utilizó una muestra no probabilística, se desarrollo a partir del estudio de caso de cinco niños que viven en la calle en alrededores del Mercado Mayorista de la ciudad de Huancayo, Perú; que fueron identificados a partir de informantes claves; quienes tenían contacto directo con niños, eran personas a quienes ellos ayudaban para ganarse el alimento diario o alguna propina, a estos informantes claves se les comunicó el objetivo de nuestro estudio y ellos mostraron su colaboración; se hizo abordajes paulatinos y progresivos en las primeras horas del día específicamente entre 5 a 6 am.

El total de intervenciones a cada niño estudiado fue entre 3 a 4 veces, en cada oportunidad se les ofrecía un incentivo que consistía en "alimentos" (frutas, sándwichs, galletas, chocolates, jugos empacados) como estrategia para poder ganar su confianza, y en ese proceso se iba recopilando información que finalmente se plasmaron en testimonios. Así mismo se realizó entrevistas a profundidad a informantes claves que ya sea por su contacto directo o por su experiencia de trabajo con este tipo de población contribuyeron en la investigación; estas entrevistas a profundidad fueron aplicadas a partir de la formulación de preguntas relacionadas al tema de investigación.

\section{RESULTADOS}

La investigación realizada ha permitido obtener los siguientes hallazgos: a nivel de la Provincia de Huancayo existe un promedio de 40 niños y adolescentes que se encuentran en situación de calle; distribuidos en tres grupos uno de ellos que se desplaza desde el Cerrito de La Libertad; bordeando Ocopilla y el Mercado Mayorista hasta llegar a Chilca y desde allí hacen su retorno por la misma ruta; ellos se dedican a robar; un grupo de niños se queda solo por alrededores del Mercado Mayorista y se dedican a mendigar o hacer algunos mandados de los comerciantes a cambio de alimentos y por las noches duermen en el sector de venta de papas, que es el área más oculta; y otro tercer grupo que deambula por el 
centro de Huancayo, ellos trabajan lustrando zapatos y roban por las noches se quedan a dormir en hostales ubicados en la Calle Calixto y Calle Marañón o alrededores.

La investigación ha permitido afirmar la siguiente hipótesis: "los factores de la dinámica familiar de los niños y niñas en situación de calle en alrededores del Mercado Mayorista que determinan su expulsión del seno familiar son:

1. La desestructuración en su cohesión familiar a partir de la ruptura conyugal sea por muerte o abandono hechos que hacen que surjan dificultades en el proceso de adaptación a los cambios. A esto se suma dos características: la migración y las experiencias de violencia experimentadas por los niños al interior de sus familias.

2. La existencia de inadecuados niveles de comunicación familiar por la incompatibilidad de caracteres entre padres e hijos; donde los hijos sienten que no son comprendidos por sus padres.

3. Y la existencia de un negativo clima emocional afectivo por escasas expresiones de afecto y estimulo entre padres e hijos.

La investigación tuvo un carácter cualitativo, se desarrolló a partir del estudio de casos de 05 niños que viven en la calle en alrededores del Mercado Mayorista de Huancayo plasmados en sus testimonios:

1. Steven, es un niño de 11 años, lo ubicamos en el sector de comidas por un informante clave, dejó su pueblo a causa de la muerte de su madre, su tía le trae a Huancayo pero le hace trabajar, el deja la casa de su tía, porque ella se apodera de su dinero, sobrevive lavando platos, y por un plato de comida duerme en el sector de venta de papas del Mercado Mayorista.

2. Pablo, es un niño de 12 años, lo ubicamos en el sector de venta de papas por un informante clave, dejó su pueblo a causa de la situación económica de su familia, sus padres deciden enviarlo a Huancayo para que estudie y trabaje con una tía; pero, en casa de su tía es víctima de maltratos, las constantes peleas de la ella con su esposo e hijos y el hecho que la tía se apoderara del dinero que ganaba trabajando hizo que él decidiera dejar la casa y quedarse a vivir en la calle, sobrevive pidiendo limosna por las noches cerca al supermercado Plaza VEA. Pablo duerme también en el sector de venta de papas del Mercado Mayorista.

3. Efraín, es un niño de 12 años, lo ubicamos en la Av. Ferrocarril en la intersecciones del Jr. Cajamarca y Jr. Piura, dejó su casa por los constantes problemas familiares su padre abandonó su hogar para irse a vivir con otra mujer y su madre se dedicaba a beber alcohol, él no tenía quien lo cuidara y/o protegiera, en su casa no habían normas que respetar sobrevive pidiendo limosna a veces ayudando a los vendedores de salchipapas que se ubican en las noches en la Av. Ferrocarril; duerme en alrededores del Mercado Mayorista no tiene un lugar fijo, y es amigo de Pablo.

4. Pedro, es un niño de 12 años, lo ubicamos en el sector de descarga junto a los estibadores, dejó su casa porque su papá lo maltrataba mucho; antes de dejar su casa vendía caramelos para ayudar en la economía de su hogar, su papá se apoderaba de las ventas que realizaba, cansado de tanto abuso decidió dejar su casa, actualmente sobrevive ayudando a los estibadores a guiarlos cuando descargan y se gana unos centavos para comer, no quiere vender porque dice que con la cara y ropa que tiene nadie le compraría sus caramelos.

5. Elías, es un niño de 10 años, lo ubicamos en el sector de frutas, dejó su casa porque su papá hace más de un año con el pretexto de "ir a trabajar lejos" los abandonó, su madre sale al mercado a buscar trabajo y él le sigue, lo ubicamos ayudando a los vendedores de fruta a descargar las cajas de fruta por su trabajo a veces le regalan frutas o le dan alguna propina. Piensa que en la calle se sufre, pero se aprende, dice "aquí todo es chévere, voy al "pimbol" a jugar con los otros, luego de ayudar a las fruteras". 


\section{DISCUSIÓN}

Contrastando los hallazgos de la investigación afirmamos lo siguiente:

1. Es la desestructuración de la cohesión familiar a partir de la ruptura conyugal sea por muerte - abandono hechos que hacen que surjan dificultades en el proceso de adaptación a los cambios, a esto se suma dos características, uno es la migración y las experiencias de violencia experimentadas por los niños al interior de sus familias.

Reforzamos nuestros hallazgos cuando Casquero (1) manifiesta que:

Las familias de los cuales proceden los niños en situación de calle muestran altos índices de violencia familiar, expresadas en el maltrato físico y psicológico, el descuido y el abandono de los padres con respecto a sus hijos, es otra característica de las familias de estos niños; padres que dejan solos a sus hijos y no les brindan una atención adecuada para un buen desarrollo físico, psicológico, emocional y social.

Así mismo Flores (2) manifiesta que:

La violencia familiar es el factor desencadenante de la salida del hogar. Sin embargo, los niños no salen de sus casas a un mundo absolutamente desconocido, tienen experiencia de vida callejera previa. El conocimiento de las calles les permite una opción, otro horizonte para sus vidas; descubren que se puede vivir sin temor y aun con alegría. Con sus amigos de la calle comparte, pero también se cuida de ellos; la solidaridad absoluta en el grupo es sólo una imagen mítica que no se correlaciona con una realidad mucho más violenta, donde el niño incluso no logra expresar todos sus sentimientos porque pueden ser entendidos como síntoma de debilidad.

Al respecto la Teoría General de Sistemas nos permite explicar este fenómeno cuando en su contenido menciona que: la realidad es observada desde dos tipos de sistemas: sistemas abiertos y sistemas cerrados; utilizamos el sistema abierto, cuando el individuo mantiene una relación permanente con su medio ambiente basado en procesos de interacción permanente; aplicado a la investigación afirmamos que el funcionamiento de una familia como un todo, no depende tanto de saber qué ocurrió tiempo atrás, ni de la personalidad individual de los miembros de la familia, sino de las reglas internas del sistema familiar, en el momento en que lo estamos observando y estas reglas determinaran su estructuración o desestructuración.

2. Existe inadecuados niveles de comunicación familiar por la incompatibilidad de caracteres entre padres e hijos; donde los hijos sienten que no son comprendidos por sus padres.

Al respecto afirmo que efectivamente el hecho que la familia experimente inadecuados niveles de comunicación, específicamente donde las órdenes están basadas en un autoritarismo puro ejercida por el jefe de hogar, así mismo la lucha de dominar y no dejarse dominar entre sus miembros hace que no exista comunicación y si la hay estos son escasos y distorsionados; los niños no confían en sus padres, no creen en ellos, observan que sus conductas son inadecuadas y por ello su palabra no es creíble y al no tener respuesta a las "órdenes" surge el maltrato o conflicto.

Con relación a lo anterior nuestros informantes claves manifiestan:

- No considero que existan niveles de comunicación; al contrario considero que la comunicación se da por dos aspecto uno por el "aspecto afectivo" que en su mayoría es la madre quien da más afecto que el padre y el otro es el "aspecto de autoridad" que principalmente es ejercida por el jefe de familia donde las normas autoritarias muchas veces no son de plena conformidad por todos los miembros de la familia y aquí cuando no hay conformidad surge el conflicto (Informante Clave № 01 Mg. Ricardo Soto Sulca Docente Universitario, con experiencia directa en el trabajo con niños y adolescentes en la Región Junín y Huancavelica)

- Existen problemas de comunicación vertical, eso se practica desde quien tiene mayor jerarquía y los hijos optan por actitudes de sumisión. (Informante Clave № 02 Lic. Pilar Miranda Huamani Coordinadora del Programa de 
Educadores de Calle - INABIF)

- Existe comunicación distorsionada; solo se comunican con gritos y golpes. (Informante Clave № 03 Lic. Liliana Naupari Samaniego Educador de Calle Programa INABIF)

- Los niveles de comunicación en el interior de la familia es difícil; cuando el chico sale a la calle aprende muchas cosas nuevas y al regresar a casa siente que el sabe más que los demás y por lo tanto no hay nada que hablar con alguien que no piensa como él y por consiguiente los niveles de comunicación se rompen por que no se ponen de acuerdo. (Informante Clave № 04 Lic. Ricardo Tinoco Aguilar Educador de Calle - Programa INABIF)

- La comunicación es de muy baja calidad por la misma situación de pobreza, no hay espacios para escuchar principalmente para ofrecer amor, afecto y ternura a los niños. (Informante Clave № 05 Enrique Jaramillo García Colaborador del MNNATSOP, Presidente de la Asociación "Niños del Río", Docente del Curso Virtual IFEJANT, Docente en la Maestría de Políticas Sociales con mención en Promoción de la Infancia UNCP Huancayo)

Todo lo descrito permite afirmar que los niveles de comunicación son muy escasos y si lo hay es distorsionado, los hijos sienten que sus padres no los comprenden y los padres sienten que sus hijos no los respetan; así mismo piensan que "comunicación es impartir órdenes y cumplirlas" se olvidan que es algo más integral va desde saber escuchar al otro, ponerse en su lugar, sentir y expresar emociones.

Al respecto Pimentel menciona que: "las familias están viviendo cambios profundos que ha significado el empeoramiento de sus condiciones de vida diaria agudizando mas los conflictos y la violencia entre ellos"; y esto afecta en las conductas de pareja como en las relaciones familiares de padres a hijos acentuándose los conflictos y peleas al interior de la familia que provocan la salida del niño a las calles (3)

Para explicar científicamente la veracidad de esta hipótesis utilizamos la Teoría del Rol que sustenta que en una persona sus características propias tienen mucha relación con el comportamiento de cada uno de los miembros al interior de la familia; por lo tanto cada miembro de la familia manifiesta comportamientos que lo ayudan a interrelacionarse unos con otros, lo cual hace que sea actor pasivo o activo dependiendo de su posición al interior de su familia.

La Teoría del Rol, nos permitirá analizar con objetividad la dinámica familiar de los niños en situación de calle sustentando nuestra hipótesis planteada donde el rol de jefes de hogar lo asumen los que desempeñan el papel de padres, y en función al rol se define sus funciones y su manera de comunicarse con los otros es así que el padre/madre es quien imparte las órdenes y en su mayoría de veces su tipo de comunicación es autoritario y vertical frente a ello los hijos no están de acuerdo con estas situaciones y es allí donde surge el conflicto.

A esto se suma la cultura donde los padres fueron criados que es muy diferente en el cual se desarrollan los niños, esta diferenciación de culturas que sustentan los estilos de crianza son diferentes y por consiguiente surge el conflicto; así mismo cuando el padre se va de casa la madre se queda sola y debe asumir el rol de padre y madre y las funciones que tiene son múltiples lo que motiva que la madre este cansada y al momento de querer escuchar a sus hijos no lo realice y no se sienta comprendida por sus hijos; ya que "cambiar de un papel a otro puede causar confusión o conflicto en el individuo".

3. Finalmente se afirma que existe un clima emocional afectivo negativo por escasas expresiones de afecto y estimulo entre padres e hijos. El hecho que la familia experimenta en su dinámica familiar un clima emocional negativo, por las escasas expresiones de afecto y estímulo entre padres e hijos; en su mayoría los padres no refuerzan las potencialidades de sus hijos con estímulos al contrario sancionan los fracasos con expresiones negativas, con expresiones de desvalorización.

Experimentan niveles de carencia afectiva, como se decía, muchas veces la fuente de afecto ya no es hacia ellos, sino hacia otros; así mismo considero que las costumbres y niveles de trasmisión generacional es 
netamente cultural y no desde una explicación biológica y/o psicológica.

El afecto, el cariño va en carencia porque la calle se vuelve atractivo, al chico le da opciones múltiples; "la calle no tiene paredes"; no hay límites; y su casa se vuelve más fría, no hay televisión, no hay comida.

Los niños de situación de calle tienen la parte afectiva y emocional muy frágil, ante cualquier tipo de problema se derrumban y se dan al abandono, esta situación les hace proclives al consumo de pegamentos, primero "terokal" luego drogas, y así siguen para olvidar sus penas.

Al respecto Alor señala que los diversos problemas como maltrato y discriminación que se suscitan al interior de la familia, desencadena en abandono moral y material del niño incitando que éstos busquen espacios de compensación afectiva y en su mayoría lo encuentran en sus pares, esta experiencia lo motiva salir fuera del hogar y permanecer por un largo tiempo en la calle (4)

En la Teoría General de la Acción Social de Parson está circunscrito en función al actor, en la investigación los actores son los niños y niñas que se sitúan en la calle por un periodo prolongado de tiempo, y se definen como el o los individuos que son motivados en busca de metas para satisfacer aquello que no satisficieron en su hogar y como fin último es su tranquilidad que aparentemente lo encuentran en la calle. Alguno de los factores que Parson considera que intervienen en la acción, es la situación que ejerce su influencia para alcanzar sus fines, el actor debe poseer medios y posteriormente superar condiciones que constituyen obstáculos para el logro de sus propósitos.

Esta situación es definida como negativa donde confluyen una serie de factores que determinan "carencias" y por ende la insatisfacción de estos factores, los medios que posen los niños y niñas cuando toman la decisión de salir de su hogar únicamente es su fuerza de voluntad, en algunos casos su estado de salud mental, la amistad de otros niños que previamente lo motivaron para tomar la decisión final; así mismo la situación también se define en los diversos obstáculos que encuentra el niño o niña al quedarse en la calle lo primero será enfrentarse a diversos peligros como robos, atropellos, etc.

\section{REFERENCIAS BIBLIOGRAFCAS}

1. Casquero R. La realidad de las niñas, niños y adolescentes en situación de calle una aproximación cualitativa. Centro de Información y Educación para la prevención de abuso de Drogas Lima: CEDRO; 2004.

2. Flores I. Los niños y el trabajo. Niños en estrategias de sobrevivencia. Revista Pediátrica Asociación de Médicos Residentes del Instituto Nacional del Niño. 2000; 3(2): 29-32

3. Pimentel C. Las Familias y sus Problemas en los Sectores Urbanos Pobres. Lima: CECOSAM; 1996.

4. Alor H. Proyecto Amigos de los niños de la calle Perú. Publicación del Instituto Internacional de Planteamiento de la Educación. Lima: UNESCO; 1999.

Correo electrónico:

cecyaass@hotmail.com 